\title{
Increasing Cognitive Assessment Rates in an Internal Medicine Clinic: A Quality Improvement Project
}

\author{
Cara Levin, $M D^{7}$ (D) Emily Morgan, $\mathrm{MD}^{1,2}$, Steven Kassakian, $\mathrm{MD}, \mathrm{MS}^{1,2,3}$, \\ Faiza Khan, $\mathrm{MBBS}, \mathrm{MB}^{4}$, Elizabeth Eckstrom, $\mathrm{MD}, \mathrm{MPH}^{1,2}$, and Christopher Terndrup, $\mathrm{MD}^{1}$
}

'Department of Medicine, Oregon Health \& Science University, Portland, OR, USA; 'Division of General Internal Medicine and Geriatrics, Oregon Health \& Science University, Portland, OR, USA; ${ }^{3}$ Medical Informatics and Clinical Epidemiology, Oregon Health \& Science University, Portland, OR, USA; ${ }^{4}$ Business Intelligence \& Advanced Analytics, Oregon Health \& Science University, Portland, OR, USA.

J Gen Intern Med 36(1):227-9

DOI: $10.1007 / \mathrm{s} 11606-019-05628-\mathrm{w}$

(c) Society of General Internal Medicine 2020

\section{INTRODUCTION}

In 2018, an estimated 5.7 million people were living with Alzheimer's dementia; this number is projected to grow to nearly 13.8 million by 2050 , with costs exceeding 1.1 trillion dollars. ${ }^{1}$ Clinical care enhancements and quality measures for management of dementia are critical to the health of older adults in the US. ${ }^{2}$ With variable and unpredictable rates of decline, ${ }^{3}$ having updated cognitive assessments allows the care team to target treatment goals, monitor interventions, and plan care-level transitions.

Our project aimed to promote regular cognitive assessments, as recommended in the 2018 "Dementia Work Group" quality measures report ${ }^{3}$ and supported as a Comprehensive Primary Care Plus $(\mathrm{CPC}+)$ Center for Medicare and Medicaid (CMS) metric. The "Dementia: Cognitive Assessment" (CMS149, version 7 available here) aims to increase the percentage of patients, "with a diagnosis of dementia for whom an assessment of cognition is performed and the results reviewed at least once within a 12 month period. ${ }^{44} \mathrm{We}$ sought to improve performance in a sustainable way that used existing resources and avoided excessive provider burden.

\section{METHODS}

We collaborated with our Clinical Informatics department to standardize data capture, which allowed us to use

Received December 4, 2019

Accepted December 19, 2019

Published online January 10, 2020 flowsheets within the EHR for easier identification of cognitive assessment tools. Next, we created an automatic reminder for any patient with a diagnosis of dementia or MCI. Patient reminders are a specific type of clinical decision support (CDS) and have been shown to improve adherence to preventative performance measures. ${ }^{5} \mathrm{We}$ incorporated the cognitive assessment performance into standard patient rooming workflows for improved reliability and durability. Medical assistants are prompted by the CDS to provide paper forms for cognitive assessment during the visit; we piloted this integration on a small scale and found it to be feasible prior to clinic-wide dissemination.

To determine an accurate denominator, we generated a list of all patients with any memory-related diagnosis, including over 200 non-specific codes such as memory loss and cognitive decline. Providers received this list, along with information from last assessment, current problem list, medications, laboratory, and imaging results. They were asked to either document a specific diagnosis code, schedule an appointment for further assessment, or refer for evaluation with a geriatrician. The provider could then update the patient reminder for cognitive screening as complete or contact the scheduler to follow up with any patient due for assessment. Patients with advanced dementia were excluded given poor utility of ongoing assessment.

\section{RESULTS}

Baseline data showed that $27.9 \%$ of patients with a diagnosis of dementia had a cognitive assessment within the last 12 months. The CPC+ target is $55 \%$; we created an attainable goal of $50 \%$ over 6 months. We tracked progress using an EHR dashboard with $\mathrm{CPC}+$ metrics 


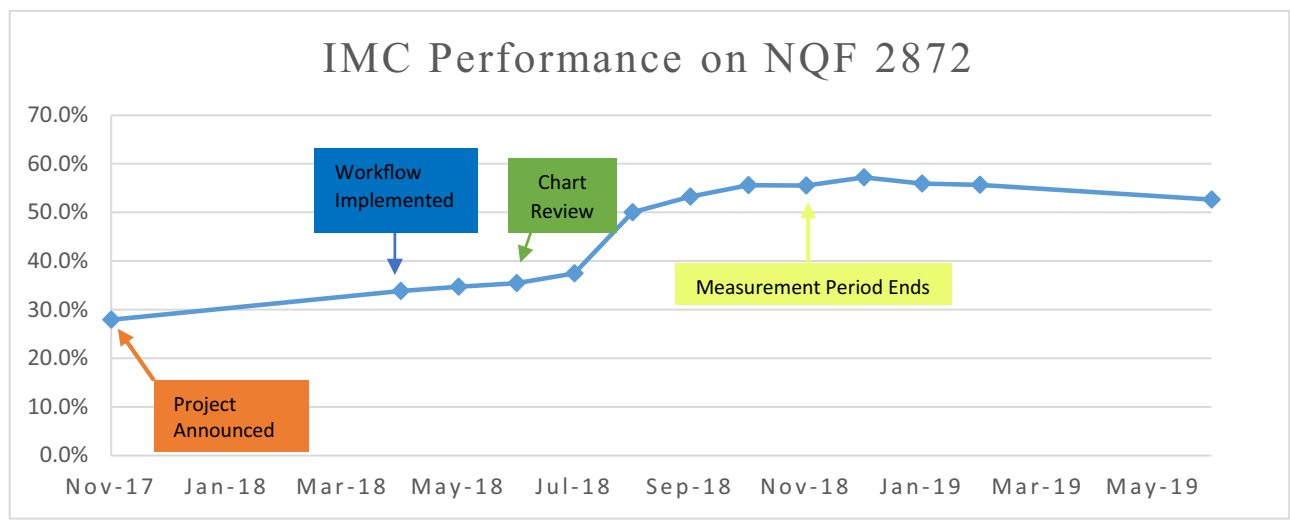

Fig. 1 Performance on cognitive assessment metric over time.

and saw significant improvement in our clinic performance in adherence this metric to $55.5 \%$ (Fig. 1).

We used a non-equivalent dependent control variable to help control for secular trends, ${ }^{6}$ the NQF 0418, Screening for Depression and Follow-up Plan, which involves a similar workflow. We also compared our performance to a similar primary care clinic within our healthcare system as an untreated control group for CMS149. There was no concurrent change in either control variable (Fig. 2).

\section{DISCUSSION}

This quality improvement project was successful in increasing the percentage of our patients with dementia who had annual cognitive assessments. This highlights the importance of utilizing CDS tools within the EHR and coupling those with workflow changes that can easily be monitored and maintained.

Limitations include a lack of "gold standard" in determining if providers accurately diagnose patients with dementia. The push to clarify specific diagnoses on patients had no ongoing workflow associated with it and may require an annual reminder for sustainability. Next steps include further examination of how certain EHR tools could improve the care of patients with dementia, along with targeted evaluation and treatment plans matched to the level of cognitive decline. Lastly, improved case finding in primary care would benefit our aging population.

We implemented this project in our clinic to improve performance on the important dementia care metric of annual

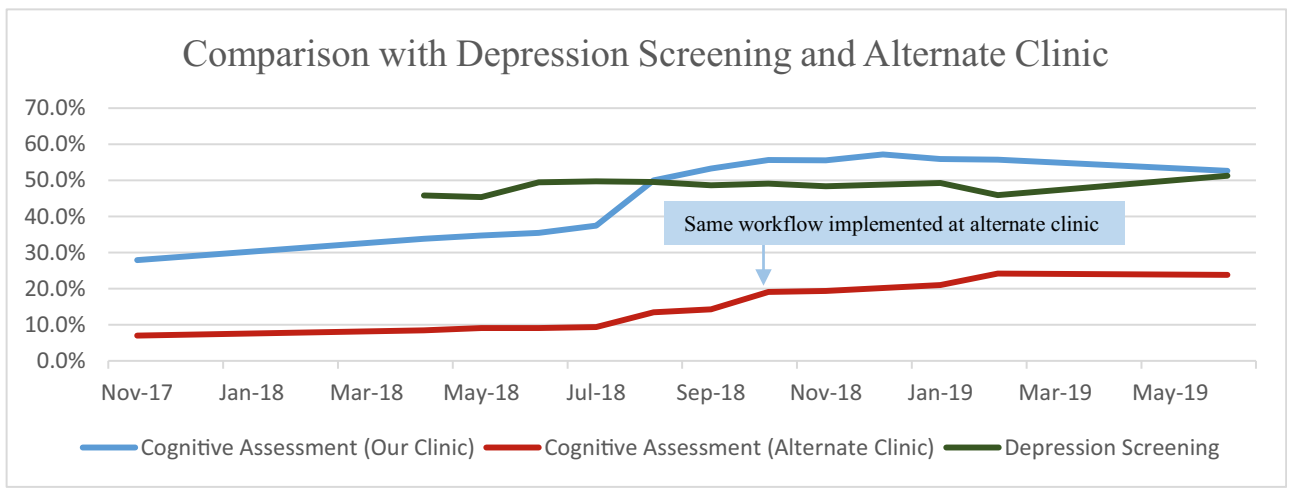

Fig. 2 Comparison of performance on cognitive assessment metric to depression screening and alternate clinic. 
cognitive assessments. By integrating a user-friendly panel review process, and integrating action items into existing workflows, we were able to exceed our initial performance goal.

Corresponding Author: Cara Levin, MD; Department of Medicine Oregon Health \& Science University, 3181 SW Sam Jackson Park Rd, Portland, OR 97239, USA (e-mail: colevin@gmail.com).

\section{Compliance with Ethical Standards:}

Conflict of Interest: The authors of this article have no conflicts of interest to report.

\section{REFERENCES}

1. Alzheimer's Association. (2018). 2018 Alzheimer's disease facts and figures. Alzheimers Dement, 14(3), 367-429
2. Odenheimer, G., Borson, S., Sanders, A. E., Swain-Eng, R. J., Kyomen, H. H., Tierney, S., ... \& Johnson, J. (2013). Quality improvement in neurology: dementia management quality measures. Neurology, 81(17), 1545-1549

3. Mitchell SL, Teno JM, Kiely DK, et al. The clinical course of advanced dementia. N Engl J Med 2009;361:1529-1538.

4. eCQI Resource Center. "Dementia: Cognitive Assessment. Accessed August 28, 2019 at https://ecqi.healthit.gov/ecqm/ep/2019/cms149v7.

5. Loo, T. S., Davis, R. B., Lipsitz, L. A., Irish, J., Bates, C. K., Agarwal, K., ... \& Hamel, M. B. (2011). Electronic medical record reminders and panel management to improve primary care of elderly patients. Arch Intern Med, 171(17), 1552-1558.

6. Harris, A. D., McGregor, J. C., Perencevich, E. N., Furuno, J. P., Zhu, J. Peterson, D. E., \& Finkelstein, J. (2006). The use and interpretation of quasi-experimental studies in medical informatics. J Am Med Inform Assoc, 13(1), 16-23.

Publisher's Note: Springer Nature remains neutral with regard to jurisdictional claims in published maps and institutional affiliations. 